\title{
Categorizing Potential Planetary Mission Types
}

A white paper submitted to the Planetary Science Decadal Survey

Graeme Hardin

WA Astronomy Support Foundation

+61422989118 (UTC+08:00)

graemehardin@gmail.com 


\section{Categorizing Potential Planetary Mission Types}

The following brief white paper submission to the Decadal Survey has just one point of emphasis.

Even as a geologist with a background in the geochemistry of Earth's Archaean, whose submission might therefore be expected to emphasise the importance of related planetary exploration questions, it is instead easy to recognize and acknowledge that just one particular question, which is resolvable by planetary exploration and potentially determinable within the Decadal Survey timeframe, dwarfs all other questions which could be targeted in that timeframe, and dwarfs them by orders of magnitude.

For example, questions regarding the interiors of Neptune and Uranus absolutely pale into insignificance compared to the question of whether life exists, or ever existed, within our solar system beyond Earth. [There is no intention to disparage Neptune or Uranus research by using this example: the areas of research mentioned are simply randomly chosen -- but major - examples of nonastrobiological topics which are of similar scientific magnitude to any other important nonastrobiological questions which might be answerable in forthcoming planetary missions.]

Accordingly, this submission urges the Decadal Survey Committee members to recognize the overarching truth of that fact - that the astrobiology question absolutely dwarfs all others - and to factor that truth into all of their deliberations and recommendations.

As a major example of how that could be done, the following is highly relevant. Due to the very high sophistication of any planetary-mission instruments required to make progress on the astrobiological question, it is absolutely inevitable that vast amounts of data which can be used to illuminate many unrelated questions will be acquired during any astrobiology-focused mission. And, since it is a highly subjective task to rank the relative importance of the infinite number of non-astrobiological questions, it is just as fair to simply allow light to be shed on the non-astrobiological questions by a purely serendipitous approach - ie by talented investigators making "by-catch" use of the massive amounts of data acquired during astrobiology missions.

Therefore, all of the following five mission types should be ranked by the Decadal Survey Committee as having by far the highest priority in this Decadal Survey (in addition to Dragonfly and Europa Clipper, and with absolutely no internal order of precedence implied by the order below), and well ahead of all non-astrobiological missions:

- Mars sample return

- Sample return, and other investigations, of the unidentified UV absorbers in the Venus atmosphere

- Plume-sampling and other astrobiological investigations of Enceladus

- Astrobiological-focused lander on Ceres

- Astrobiological-focused lander on Europa

It is perfectly reasonable that one or more of these missions may reveal life beyond Earth, in which case the scientific discovery is orders of magnitude more important than any other conceivable discoveries by alternative (non-astrobiological) planetary missions. And even if all five missions eventually reported negative results, the value of that information would be of an almost similar magnitude, with great importance for all theories and calculations regarding the likely existence or 
otherwise of life beyond Earth (and that is without including the non-astrobiological discoveries derived from the enormous amount of inevitable by-catch data obtained on such missions.)

Accordingly, all specialists in other fields should temporarily put pet projects to one side, and accept that, for this short period of human history, all solar system planetary exploration effort should focus on the astrobiological question, with other questions to be progressed by piggybacking on these missions or by serendipitous use of the large volumes of data acquired. 